\title{
Interstellar extinction by spheroidal dust grains
}

\author{
R. Gupta ${ }^{1}$, T. Mukai ${ }^{2}$, D. B. Vaidya ${ }^{3}$, A. K. Sen ${ }^{4}$, and Y. Okada ${ }^{2}$ \\ 1 IUCAA, Post Bag 4, Ganeshkhind, Pune-411007, India \\ e-mail: rag@iucaa.ernet.in \\ 2 The Graduate School of Science and Technology, Dept. of Earth \& Planetary Sciences, Faculty of Science Kobe University, \\ Nada, Kobe 657-8501, Japan \\ ${ }^{3}$ Gujarat College, Ahmedabad-380006, India \\ ${ }^{4}$ Department of Physics, Assam University Silchar-788011, India
}

Received 17 January 2005 / Accepted 21 June 2005

\begin{abstract}
Observations of interstellar extinction and polarization indicate that the interstellar medium consists of aligned nonspherical dust grains which show variation in the interstellar extinction curve for wavelengths ranging from NIR to UV. To model the extinction and polarization, one cannot use the conventional Mie theory which assumes the grains as solid spheres. We have used a T-matrix based method for computing the extinction efficiencies of spheroidal silicate and graphite grains of different shapes (axial ratios) and sizes and used these efficiencies to evaluate the interstellar extinction curve in the wavelength range 3.4-0.1 $\mu \mathrm{m}$. A best fit linear combination of silicate and graphite grains of not very large axial ratio, fits the observed extinction curve reasonably well.

We calculate the volume extinction factor $V_{\mathrm{c}}$, which is an important parameter from the point of view of the cosmic abundance, for the spheroidal grain models that reproduce the interstellar extinction curve. We find that the shape of the grains does not affect the volume extinction factor.

Finally we have also studied the extinction and linear polarization efficiencies for aligned spheroids. The results show that the shape of grains affects the linear polarization efficiencies considerably for various orientation angles of the spheroids.
\end{abstract}

Key words. dust, extinction

\section{Introduction}

The exact solution to Maxwell's equations to calculate absorption, scattering and extinction of electromagnetic waves by homogeneous isotropic spheres is provided by Mie theory (van de Hulst 1981). Spherical grain models have been used by many authors to evaluate the interstellar extinction curve (e.g. Mathis et al. 1977; Draine \& Lee 1984). However, the spherical grains can not explain the observed interstellar polarization that accompanies interstellar extinction. Mie theory would also not be strictly applicable if the particles have anisotropic optical properties, as for example graphite. Using the method of separation of variables Asano \& Yamamoto (1975) have given the analytical solutions for homogeneous isotropic spheroidal particles. The method of separation of variables is based on expanding the incident, internal and scattered fields in vector spheroidal functions. For spheroids with large sizes, large refractive indices or absorbing particles, the system of linear equations becomes large and it becomes a difficult mathematical and numerical problem. Recently, Voshchinnikov \& Farafonov (1993) have improved the separation of variables method which is applicable to large aspect ratios. Several numerical techniques are now being in practice to obtain scattering and absorption cross sections for nonspherical particles (e.g. Bohren \& Huffman 1983; Mishchenko et al. 2000). The
T-Matrix technique is based on expanding the incident field in vector spherical wave functions (VSWFs) regular at the origin and expanding the scattered field outside a circumscribing sphere of a scatterer in VSWFs regular at far field region. The T-Matrix transforms the expansion coefficients of the incident field into those of the scattered field and can be used to compute any scattering characteristic of a nonspherical particle (for details on T-Matrix method see Mishchenko et al. 2002).

One can also use the discrete dipole approximation (DDA see viz. Draine 1988) to study the extinction properties of the nonspherical inhomogeneous particles (see Borghese et al. 2003). We have used the DDA to study the extinction properties for porous and composite particles (Vaidya et al. 1997, 1999, 2001; Papers I, II and III hereafter). However, the DDA method required considerable computer time and memory. The T-Matrix code on the other hand (Mishchenko et al. 2002) runs much faster and the results obtained can be tuned with much ease since the input parameters to the code can be adjusted and re-run in a short time. However, the T-Matrix code in its present form cannot be used for studying inhomogeneous (e.g. porous, fluffy, composite) particles (Mishchenko et al. 2002).

In Sect. 2 we present the spheroidal grain models and describe the T-Matrix method; in Sect. 3 we give the results of 
our calculations; i.e. extinction curves as functions of grain size, shape and wavelengths, the model interstellar extinction curves, and comparison of these model curves with the observed interstellar extinction curve (Savage \& Mathis 1979; Whittet 2003) and the influence of particle shape on the determination of interstellar extinction and in Sect. 4 we provide the conclusions of the present study.

\section{T matrix method and spheroidal grain models}

The T-Matrix method was first introduced by Waterman (1965) for studying electromagnetic scattering by single, homogeneous nonspherical particles. The standard scheme for computing the T-Matrix for single homogeneous scatterers in the particle reference frame is called the extended boundary condition method (EBCM) and is based on the vector Huygens principle (Waterman 1971). The general problem is to find the field scattered by an object bounded by a closed surface. The Huygens principle establishes the relationship between the incident field, the total external field (i.e. the sum of the incident and the scattered fields), and the surface field. Technically the incident and the scattered waves are expanded in regular and outgoing vector spherical wave functions (VSWFs); the concept of expanding the incident and the scattered waves in VSWFs and relating these expansions by means of T-Matrix has proved very useful. The great computational efficiency of the T-Matrix approach to study electromagnetic scattering by nonspherical particles with various shapes and sizes has found numerous applications. Several FORTRAN T-Matrix codes are available on the world wide web at http://www.giss.nasa.gov/ crmim. The codes compute the complete set of scattering characteristics, the optical cross sections, expansion coefficients, and the scattering matrix for randomly oriented and aligned particles.

In the present study the axial ratios (ratio of horizontal to rotational axes hereafter called as AR) of the spheroidal grains ranging from 0.5 to 2.0 have been taken. Please note that for prolates we have $A R<1$ and for oblates we have $A R>1$. The aspect ratio $\epsilon$ is the ratio of the larger and smaller axes as defined by Mishchenko et al. (2002) and thus both a prolate with $A R=0.5$ and an oblate with $A R=2.0$ will have the aspect ratio $\epsilon=2$. For spheres $A R=1.0$ and $\epsilon=1$.

The observed interstellar polarization requires that interstellar grains be aligned and nonspherical in shape. Hence, in addition to the calculations on random orientations (with orientation averaging) of the spheroidal grains we have also performed calculations for the aligned spheroids at several orientation angles.

\section{Results}

\subsection{Extinction properties of spheroidal grains}

One of the main objectives of the paper is to study the extinction efficiencies of the spheroidal silicate and graphite grains, obtained using the T-Matrix calculations and see the effects of shape of the grains i.e. spheres, oblates and prolates and various sizes which are typical to interstellar grain sizes i.e. 0.001 to about $0.500 \mu$. These materials (i.e. silicate and graphite) have been the ingredients for most of the spherical grain models (see for example Mathis et al. 1977; Draine \& Lee 1984; Weingartner \& Draine 2001). We present the results for the spheroidal grain models; i.e. the extinction efficiencies $Q_{\text {ext }}$ as a function of wavelength in the spectral range (3.4 to $0.1 \mu \mathrm{m}$ ).

Silicates: Fig. 1 shows the extinction efficiency $Q_{\text {ext }}$ for silicate grains for four grain sizes i.e. $0.01,0.05,0.1$ and $0.2 \mu$. The $y$-axis in these plots are in the form of an extinction efficiency ratio $Q_{\text {ext }}($ Spheroid $) / Q_{\text {ext }}($ Sphere $)$ to bring out the effect of oblates and prolates as compared to spheres.

It is seen from this figure that the grains with the same aspect ratio $\epsilon=2$ but having different axial ratios, $A R=0.5$ (Prolates) and $A R=2.0$ (Oblates), display similar extinction properties in the entire wavelength range, i.e. $3.4-0.1 \mu \mathrm{m}$. Results for the grains with other aspect ratio $\epsilon=1.33$, having an axial ratio $A R=0.75$ (Prolates) and $A R=1.33$ (Oblates) also show similar extinction property. These results on spheroids are consistent with the results reported by Mishchenko et al. (2002). It is also seen that the extinction efficiency ratio $Q_{\text {ext }}($ Spheroid $) / Q_{\text {ext }}($ Sphere $)$ for the silicate grains with aspect ratio $\epsilon=1.33$ (i.e. $A R=0.75$ and 1.33) is close to $A R=1.0$ (sphere), i.e. there is no appreciable variation in the extinction efficiency from spheres; whereas spheroidal grains with aspect ratio $\epsilon=2$ (i.e. $A R=0.5$ and 2.0), show considerable variation in the extinction from sphere i.e. the ratio $Q_{\text {ext }}($ Spheroid $) / Q_{\text {ext }}($ Sphere $)$ deviates from 1.0.

Graphites: Fig. 2 shows extinction curves for spheroidal graphite grains. For small graphite grains $(a=0.01 \mu)$ the "2175 ^" extinction feature is displayed by all the spheroidal grain models, but the peak is shifted for various axial ratios. In order to emphasize this aspect we show in Fig. 3 the extinction efficiency $Q_{\text {ext }}$ for the spheroidal graphite grains for $a=0.01$ and $0.05 \mu$ in the wavelength range $0.25-0.20 \mu \mathrm{m}$ (i.e. $4-5 \mu \mathrm{m}^{-1}$ ). These curves clearly show the shift in the extinction peak with the shape of the grains (i.e. axial ratio). For $a=0.01 \mu$ the extinction peak at $4.6 \mu \mathrm{m}^{-1}$ for spherical grains ( $A R=1$ and Mie) shifts to $4.4 \mu \mathrm{m}^{-1}$ for the spheroidal grains with $A R=2.0$. These curves also show the variation in the width of the feature. These results on the spheroidal grains indicate that the shape of the grains plays an important role in studying extinction properties and needs to be studied in more details. Voshchinnikov (1990) has also found the variation in the " $2175 \AA$ " feature with the shape of the grain. In our earlier study on the porous grains (Vaidya et al. 1997, 1999, i.e. Papers I and II) we had found the shift in the the "2175 $\AA$ " peak as well as the variation in the width of the feature for the graphite grains with porosity. Draine \& Malhotra (1993) have found the shift in the central wavelength of the feature in the coagulated graphite grains but did not find any appreciable change in the width. It is to be noted from Fig. 2 that for spheroidal graphite grains with aspect ratio $\epsilon=1.33$ (i.e. $A R=0.75$ and 1.33 ), there is no appreciable variation in the extinction from spheres. It is also seen that for spheroidal graphite grains with $a>0.05 \mu$, the extinction does not deviate much from spheres in the optical and the UV spectral range. Please note that for prolate graphite with $A R=0.5$ in Fig. 2 for $a=0.01$ and $0.05 \mu$, the T-Matrix calculation does not converge beyond $8 \mu \mathrm{m}^{-1}$. 

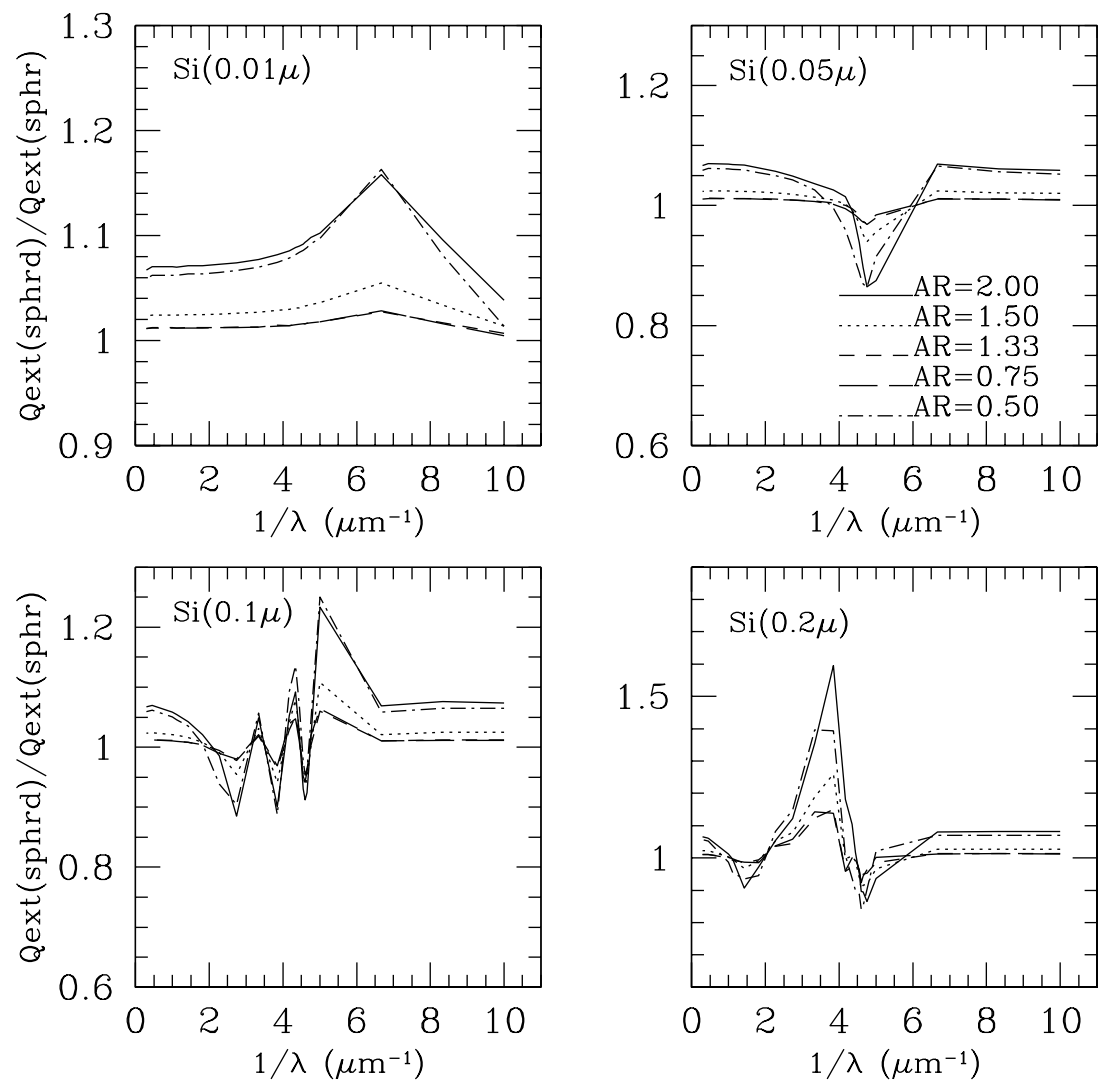

Fig. 1. Extinction efficiencies $\left(Q_{\text {ext }}(\right.$ Spheroid $) / Q_{\text {ext }}($ Sphere $\left.)\right)$ versus wavelength for randomly oriented spheroidal silicate grains for four different grain sizes and five different axial ratios.
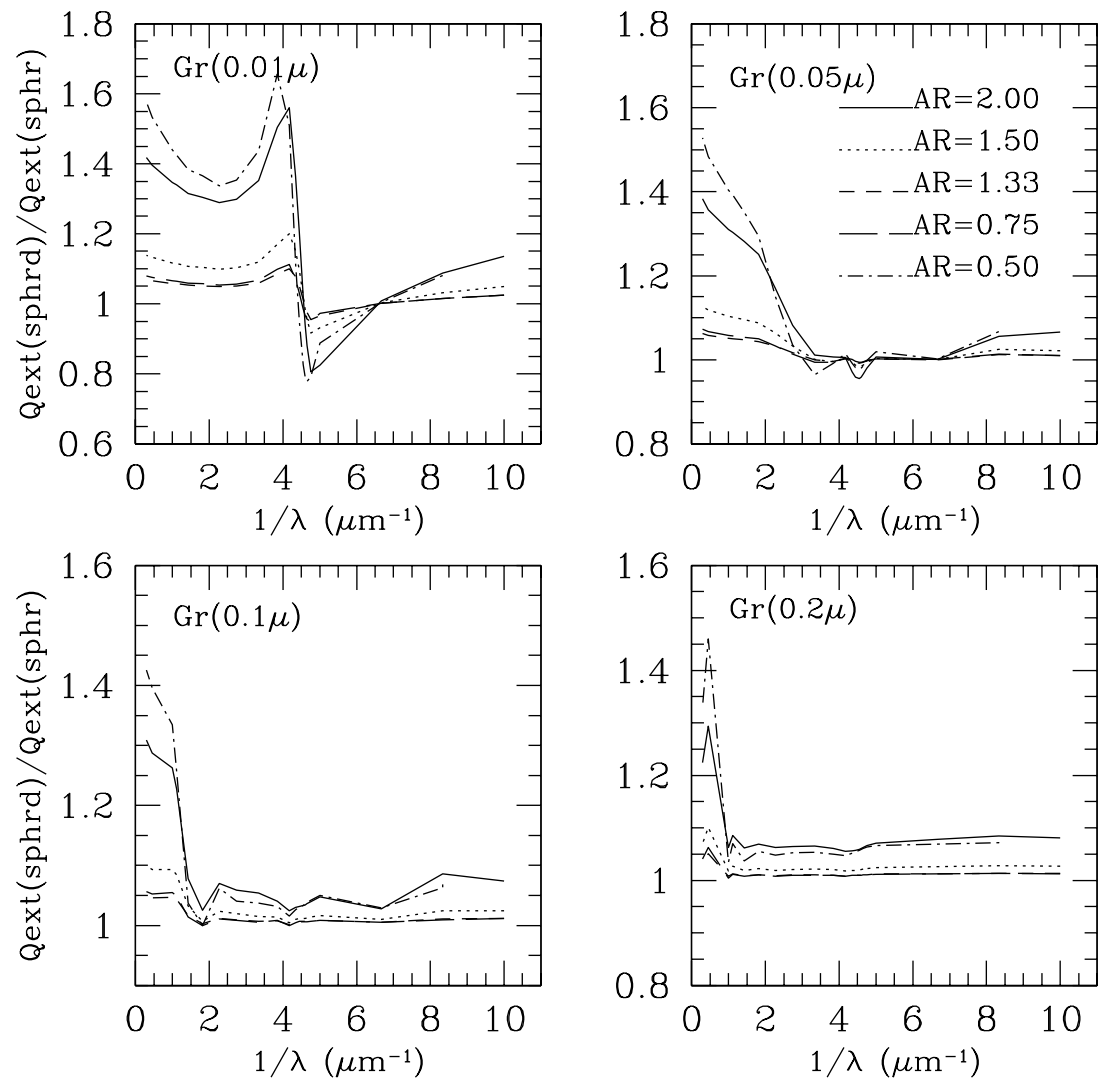

Fig. 2. Extinction efficiencies $\left(Q_{\mathrm{ext}}(\right.$ Spheroid $) / Q_{\mathrm{ext}}($ Sphere $\left.)\right)$ versus wavelength for randomly oriented spheroidal graphite grains for four different grain sizes and five different axial ratios. 

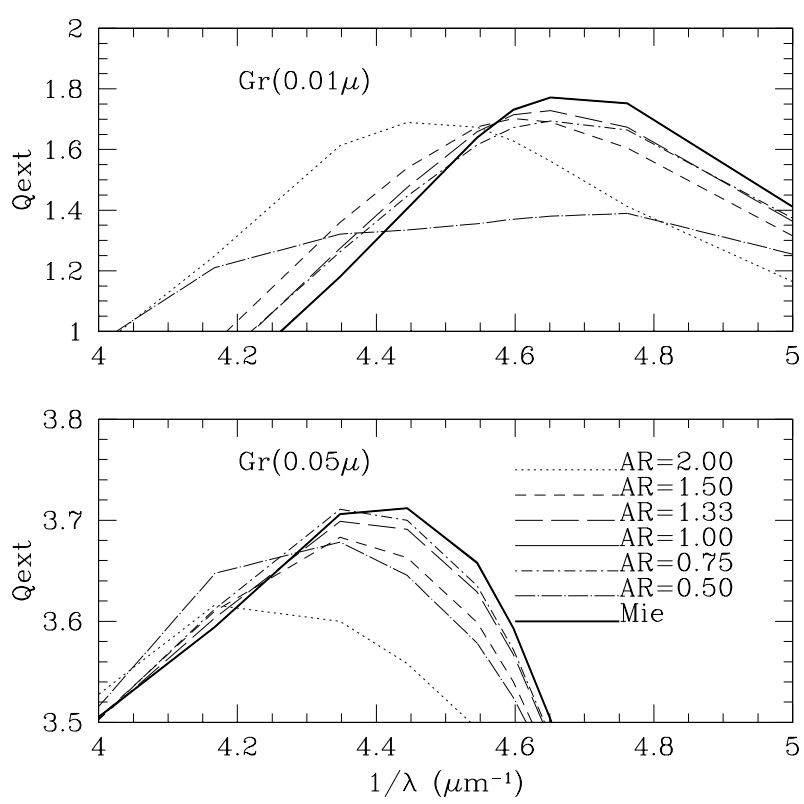

Fig. 3. Extinction efficiencies $Q_{\text {ext }}$ versus $1 / \lambda$ for randomly oriented graphite grains of sizes $a=0.01$ and $0.05 \mu$ in the UV peak region for various axial ratios. The axial ration $A R=1.00$ and Mie curves overlap each other as expected. The shift in the peak extinction is clearly seen for various axial ratios.

Extinction and polarization efficiency for aligned spheroids

As mentioned earlier the existence of interstellar polarization requires that the grains must be nonspherical and aligned (see e.g. Greenberg 1968; Wolff et al. 1993; Voshchinnikov \& Farafonov 1993). We have calculated the extinction and linear polarization for the aligned spheroidal silicate and graphite grains at several orientation angles. Figures $4 \mathrm{a}-\mathrm{d}$ show $Q_{\text {ext }}=$ $\left[Q_{\text {ext }}(\mathrm{E})+Q_{\text {ext }}(\mathrm{H})\right] / 2$ and linear polarization efficiency $\left|Q_{\text {pol }}\right|=$ $Q_{\text {ext }}(\mathrm{E})-Q_{\text {ext }}(\mathrm{H})$ for aligned (at a fixed orientation angle $\beta=45^{\circ}$ ) oblate spheroids for $a=0.1 \mu$ in the wavelength region 3.4-0.3 $\mu \mathrm{m}$; where $Q_{\text {ext }}(\mathrm{E})$ and $Q_{\text {ext }}(\mathrm{H})$ are extinction efficiency factors for the directions of the incident field vector $Q(\mathrm{E})$ and perpendicular $Q(\mathrm{H})$ to the axis of the spheroid. These results on the aligned oblates show that there is no appreciable variation in the extinction but the polarization shows considerable variation with the axial ratio. Figures $4 \mathrm{e}-\mathrm{h}$ show the extinction and linear polarization efficiency for a fixed axial ratio $A R=1.33$ but with varying orientation angles $\beta$ from 0 to $90^{\circ}$ for oblates. Here again the extinction does not show any appreciable change but the polarization does show a lot of variation. The aligned oblate silicate spheroids with $A R=1.33$ produce maximum linear polarization between 2 and $3 \mu \mathrm{m}^{-1}$, whereas the aligned graphite grains produce the maximum polarization between 1 and $2 \mu \mathrm{m}^{-1}$. These results on linear polarization are consistent with the results obtained for spheroids by Wolff et al. (1993). Figures 5a-h show extinction and linear polarization efficiencies for the aligned prolate silicate and graphite spheroidal grains. The results in Figs. 5a and 5b on aligned prolates do not show appreciable variation with the axial ratio. However, the extinction varies with the orientation angles in Figs. 5e and $5 \mathrm{f}$ for aligned prolates. Note that the polarization efficiency of oblate silicates in Figs. $4 \mathrm{c}$ and $4 \mathrm{~g}$ and prolate silicates in Figs. 5c and 5g shows considerable differences but that the rest of the curves for oblates and prolates follow similar trends.

\subsection{Interstellar extinction curve}

We use the extinction efficiencies of the silicate and graphite grains and the power law grain size distribution (i.e. $n(a) \sim a^{-3.5}$, MRN size distribution (see Mathis et al. 1977; and Papers I-III) to reproduce the average observed interstellar extinction curve (Savage \& Mathis 1979; Whittet 2003). We have evaluated the interstellar extinction curves for grain size distribution with the smaller size limit starting at $a=0.001 \mu$ and the larger size limit up to $a=1.0 \mu$. Normalized interstellar extinction curve is generated for both silicates and graphites and then combined linearly as described in our Papers I-III, to obtain the best fit with the observed extinction curve using a $\chi^{2}$ minimizing scheme.

Apart from the various sizes tried for the lower and upper limits of the size distribution; the power law index of -3.5 was also changed from -2.5 to -4.5 in steps of 0.5 and the value of -3.5 seems to be the best of the lot for obtaining best fits to the observations. Finally calculations were also repeated with different axial ratios and the best fit value of $\chi^{2}=0.012837$ was obtained with $A R=1.00$ i.e. spheres as was predicted by the MRN model. However, the spherical grains are not realistic, and besides, the observed interstellar polarization requires the interstellar grains to be non-spherical. Thus we keep the oblate spheroids with $A R=1.33$, for which the best fit $\chi^{2}$ value is 0.013210 , as the model for interstellar extinction.

Figure 6 shows the best fit $\chi^{2}$ minimized model curve along with the observed interstellar extinction curve. The top panel show the full curve in the wavelength region 3.4 to $0.1 \mu \mathrm{m}$ i.e. far UV to NIR. The bottom panel highlights the UV bump region. The best model curve thus consists of a size distribution $a=0.005-0.250 \mu$ in steps of $0.005 \mu$ and with spheroids with $A R=1.33$. The observed points (shown as filled square dots) have been evaluated at wavelength steps of $50 \AA$ by interpolation since the original data (Savage \& Mathis 1979; Whittet 2003) has much fewer wavelength points though it covers this large range. Similarly the T-Matrix model calculations were specially performed at this high wavelength resolution to provide smoother interstellar curves. Such calculations are feasible with T-Matrix due to its intrinsic property for fast computations.

\subsection{Volume extinction factor and abundance constraints}

An important parameter from the point of view of cosmic abundance is the volume extinction factor $V_{\mathrm{c}}$ which is defined as the ratio of total volume of the grains to the total extinction at a given wavelength i.e. $\sum V / \sum C_{\text {ext }}(\lambda)$ (see for example, Greenberg 1968; Vaidya et al. 1984). $V_{c}$ directly determines the amount of material required to render the level of extinction at a specific wavelength. Table 1 shows the volume extinction 
OBLATES $\mathrm{a}=0.1 \mu$
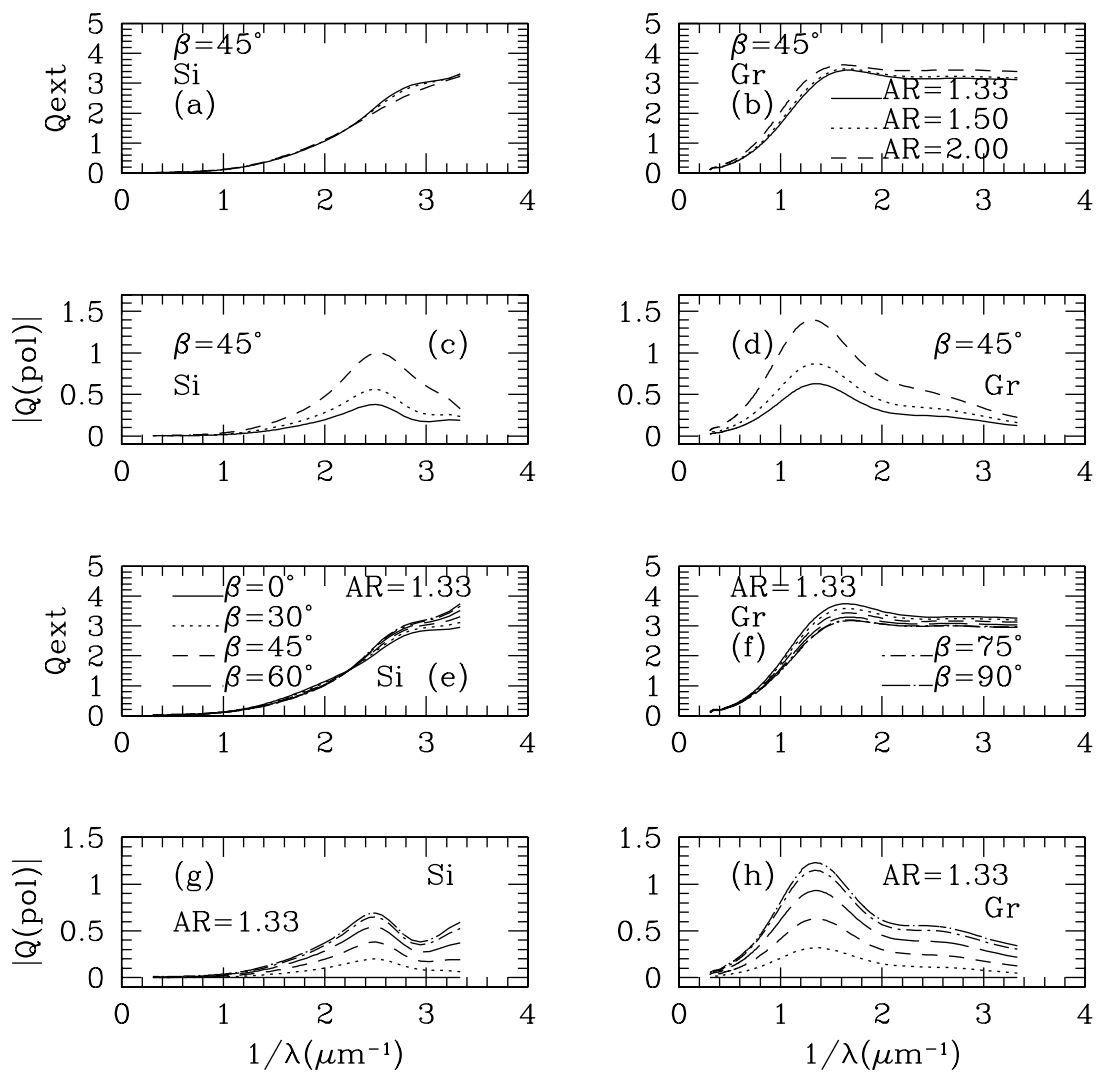

Fig. 4. Extinction $Q_{\text {ext }}$ and linear polarization efficiencies versus $1 / \lambda$ for aligned oblate spheroids (silicates and graphites with grain size $a=0.1 \mu)$. The panels $(\mathbf{a}),(\mathbf{b}),(\mathbf{c})$ and $(\mathbf{d})$ are for a fixed orientation $\left(\beta=45^{\circ}\right)$ and various axial ratios AR of the aligned spheroids. The panels $(\mathbf{e}),(\mathbf{f}),(\mathbf{g})$ and $(\mathbf{h})$ are for a fixed axial ratio $A R=1.33$ but with various orientation angles $\beta$.

Table 1. Volume extinction factor $V_{\mathrm{c}}$ for spheroidal grains.

\begin{tabular}{lccc}
\hline \hline Shape & Axial ratio & Silicate & Graphite \\
\hline Oblate & 1.33 & 0.266 & 0.102 \\
& 1.50 & 0.267 & 0.100 \\
& 2.00 & 0.271 & 0.092 \\
\hline Sphere & 1.00 & 0.265 & 0.111 \\
\hline Prolates & 0.75 & 0.266 & 0.103 \\
& 0.50 & 0.272 & 0.093 \\
\hline
\end{tabular}

factors $V_{\mathrm{c}}$ at $\lambda=0.55 \mu \mathrm{m}$ for the spheroidal grain models that reproduce the observed interstellar extinction (Fig. 6).

Results in Table 1 indicate that the variation in $V_{\mathrm{c}}$ due to shape (oblates or prolates) for both silicate and graphite grains is less than $10 \%$, hence the atomic-abundance constraints based on values of $V_{c}$ for either spheres or spheroids of each material would be identical. These results showing effect of shapes on volume extinction factor are consistent with the results obtained by Greenberg (1978).

\section{Summary and conclusions}

The extinction efficiencies for prolates, oblates and spheres are obtained using the T-Matrix calculations for silicate and graphite grains (of different sizes) in the wavelength range of $3.4-0.1 \mu \mathrm{m}$. The study brings out the effect of the shape of the grains on the extinction and linear polarization. Our results show that the shape of the grain plays an important role in determining the extinction properties of the grains and requires a more detailed study with other shapes and axial ratios. The spheroids with same aspect ratios $(\epsilon)$ tend to show similar deviations in $Q_{\text {ext }}$ values when compared with the $Q_{\text {ext }}$ values of spheres and this phenomenon has been noted by Mishchenko et al. (2002).

The $Q_{\text {ext }}$ computations were used to model the interstellar extinction and then compared with the observed curve. Best fits are obtained for grain size distribution $a=0.005-0.250 \mu$ in steps of $0.005 \mu$ (with the power law exponent value of -3.5 ) with spheroidal grains having axial ratio of $A R=1.33$.

The bare graphite/silicate grain model which we have used in the present study is consistent with observations of interstellar extinction. The volume extinction factors determined for the spheroidal grain models do not show appreciable variation from spherical models, so the abundance constraints for spheroidal grains and spheres would be identical. It should be noted the bare graphite/silicate grain model requires higher abundances of carbon and silicon than are recently available values for the interstellar medium (Mathis 2000). The real 

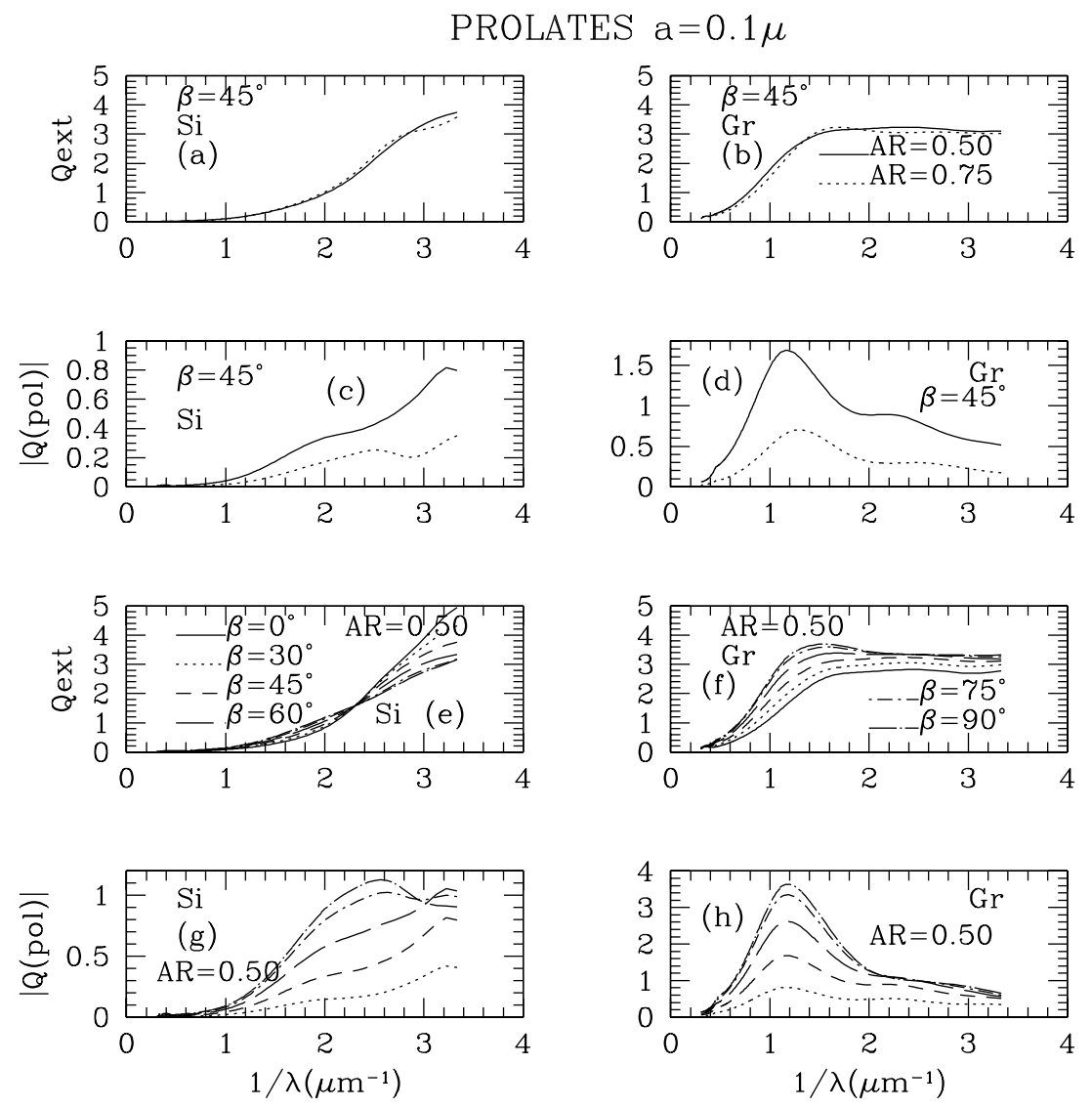

Fig. 5. Extinction $Q_{\mathrm{ext}}$ and linear polarization efficiencies versus $1 / \lambda$ for aligned prolate spheroids (silicates and graphites with grain size $a=0.1 \mu)$. The panels $(\mathbf{a}),(\mathbf{b}),(\mathbf{c})$ and $(\mathbf{d})$ are for a fixed orientation $\left(\beta=45^{\circ}\right)$ and various axial ratios $A R$ of the aligned spheroids. The panels $(\mathbf{e}),(\mathbf{f}),(\mathbf{g})$ and $(\mathbf{h})$ are for a fixed axial ratio $A R=0.50$ but with various orientation angles $\beta$.
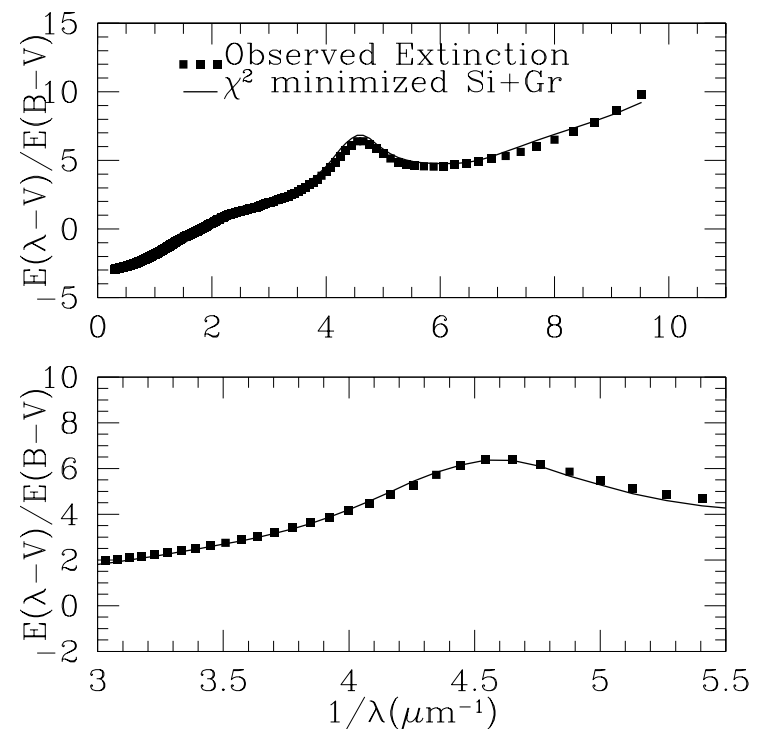

Fig. 6. The top panel shows the comparison of observed interstellar extinction curve with the best fit model combination curve for spheroidal silicate and graphite grains $(A R=1.33)$ in the wavelength region of 3.4 to $0.1 \mu \mathrm{m}$ using a grain size distribution of $0.005-0.250 \mu$ in steps of $0.005 \mu$. This plot has a wavelength resolution of $50 \AA$ for the model values and also for the observed values though the observed values are available at much lower resolution and at much fewer wavelength points. The lower panel highlights the UV bump region of the interstellar curve fitting. interstellar grains might be more complex (composite, porous; see e.g. Mathis 1996; Vaidya et al. 2001).

The T-Matrix method to study the optical properties of the spheroidal silicate and graphite grains is a new approach and there is enough scope for varying the grain shape, size and composition for obtaining better model fits to the observations. Recently composite grains have been used by Moreno et al. (2003) to model Comet Hale-Bopp dust grains. Petrova et al. (2000) have used modified version of T-Matrix to study grains and have applied these results to model cometary grains.

Acknowledgements. The authors wish to thank Mike Mishchenko for the T-Matrix code. The comments from the referee Dr. N. Voshchinnikov were extremely useful to improve the quality of the paper. The authors also wish to thank DST, New Delhi, India and the Indo-Japan Co-operative Science Programme operated by JSPS, Tokyo, Japan for providing funds to RG and AKS for this collaboration.

\section{References}

Asano, S., \& Yamamoto, G. 1975, Appl. Opt., 14, 29

Bohren, C. F., \& Huffman, D. R. 1983, in Absorption and Scattering of Light by Small Particles (New York: John Wiley)

Borghese, F., Denti, P., \& Saija, R. 2003, in Scattering from Model Nonspherical Particles: Theory and Applications to Environmental Physics (Berlin: Springer), 224 
Draine, B. T. 1988, ApJ, 333, 848

Draine, B. T., \& Lee, H. M. 1984, ApJ, 285, 89

Draine, B. T., \& Malhotra, S. 1993, ApJ, 424, 682

Greenberg, J. M. 1968, in Nebulae and Interstellar Matter, ed. B. M. Middlehurst, \& L. H. Aller (Chicago Univ. Press), 221

Greenberg, J. M. 1978, in Cosmic Dust, ed. J. A. M. McDonnell (New York: John Wiley), 187

van de Hulst, H. C. 1981, in Light Scattering by Small Particles (N.Y.: Dover)

Mathis, J. S. 1996, ApJ, 472, 643

Mathis, J. S. 2000, J. G. R., 105, A5, 10269

Mathis, J. S., Rumpl, W., \& Nordsieck, K. H. 1977, ApJ, 217, 425

Mishchenko, M. I., Hovenier, J. W., \& Travis, L. D. 2000, in Light Scattering by Nonspherical Particles: Theory, Measurements, and Applications (San Diego: Academic Press)

Mishchenko, M. I., Travis, L. D., \& Lacis, A. A. 2002, in Scattering, Absorption, and Emission of Light by Small Particles (Cambridge, UK: CUP)
Moreno, F., Munoz, O., Vilaplana, R., \& Molina, A. 2003, ApJ, 595, 522

Petrova, E. V., Jockers, K., \& Kiselev, N. N. 2000, Icarus, 148, 526

Savage, B. D., \& Mathis, J. S. 1979, ARA\&A, 17, 73

Vaidya, D. B., Bhatt, H. C., \& Desai, J. N. 1984, Ap\&SS, 104, 323

Vaidya, D. B., \& Gupta, R. 1997, A\&A, 328, 634 (Paper I)

Vaidya, D. B., \& Gupta, R. 1999, A\&A, 348, 594 (Paper II)

Vaidya, D. B., Gupta, R., Dobbie, J. S., \& Chylek, P. 2001, A\&A, 375, 584 (Paper III)

Voshchinnikov, N. V. 1990, Sov. Astron. Lett., 16(3), 215

Voshchinnikov, N. V., \& Farafonov, V. G. 1993, Ap\&SS, 204, 19

Waterman, P. C. 1965, Proc. IEEE, 53, 805

Waterman, P. C. 1971, Phys. Rev. D, 3, 825

Weingartner, J. C., \& Draine, B. T. 2001, ApJ, 548, 296

Whittet, D. C. B. 2003, in Dust in the Galactic Environment, 76, 2nd edn. (UK: IOP Publishing Ltd.)

Wolff, M. J., Clayton, G. C., \& Meade, M. B. 1993, ApJ, 403, 722 